\title{
An Energy Conservative Wireless Sensor Network Model for Object Tracking
}

\author{
Gokcer Peynirci \\ Institute of Applied Sciences, \\ Yasar University, Izmir, Turkey \\ Email: \\ gokcer.peynirci1@stu.yasar.edu.tr
}

\author{
Ilker Korkmaz \\ Dept. of Computer Engineering, \\ Izmir University of Economics, \\ Izmir, Turkey \\ Email: ilker.korkmaz@ieu.edu.tr
}

\author{
Muharrem Gurgen \\ Siemens Gebze R\&D Center, \\ Kocaeli, Turkey \\ Email: \\ muharrem.gurgen@siemens.com
}

\begin{abstract}
This study aims to find the relationship between energy consumption level and object tracking success in an object tracking sensor network (OTSN). Convenient use of energy proposes a great challenge for wireless sensor network (WSN) design and the balance between successful object tracking and low energy consumption is a tight one. To address this issue, we propose a new network operation scheme for object tracking, implement this scheme in Network Simulator 2 (ns-2) and present the obtained results of the conducted simulation experiments. The simulation results show that the proposed method can be used to track objects in a WSN network in an energy conservative manner.
\end{abstract}

\section{INTRODUCTION}

$\mathrm{W}$ IRELESS Sensor Networks (WSN) are mainly composed of a large number of sensor nodes as a network structure whose nodes are limited in terms of memory, processor and power resources. Nodes that are distributed in the environment can communicate unrestrained in short distances and adapt to its environment. By communicating with each other, they compose a variable topology layout and take form of a network themselves [1].

A sensor network provides a set of high level information processing tasks such as event detection, environmental monitoring, object tracking, or classification [2]. Sensor networks today have numerous application areas including health, military, home and various commercial applications [1]. Object tracking in WSNs have become one of the killer applications in this aspect. Although achieving object tracking using WSNs may be relatively hard depending on the object's speed, size, sensors' quality, and also the environmental conditions, it may become widespread with the right technology and methodologies in place. As a top view of the general solution to object tracking problem, a group of sensor nodes can be utilized to sense the environment for an object's location and track it along its journey in a predefined area. Meanwhile, knowing that the most critical constraint of a WSN is its lifetime, we see any study devoted to increasing it as well worth the effort.

During object tracking, a key factor is minimising energy consumption while making sure that the object is monitored. A number of metrics can be monitored to determine the energy consumption such as average number of active sensors during network operation and average length of operation per sensor node. These two provide a good performance assessment in determining energy efficiency of a WSN. There are also some other important metrics that help us determine whether an object tracking application is successful. We need to make sure the time period between the moment an object enters a monitored area and the moment it is detected by the network is kept at a minimal. Another metric is the precision of the object tracking data. This can mainly depend on the following two factors: the localization method in use and the schema used within the network for the tracking the object.

A WSN is quite an ad-hoc type of network which is built for a specific aim. The network should be designed and built with this aim in mind, in our case object tracking, the protocols and methods utilized should be in line with this aim.

WSNs vary in the way they are designed, the equipment used and ultimate goal of operation. Moreover, the way they are deployed to the area, routing protocol used or prediction algorithm used may make the difference between success and failure in object tracking scenarios. Each of these variables can affect the performance of the particular network application in question.

Some researchers [3]-[6] chose to tackle the problem of energy efficiency in object tracking in WSNs from a prediction based point of view. They based their hypothesis upon the fact that if we can predict the object's location for a given period of time, this enables us to activate only the minimal set of sensors thereby minimising energy consumption of each node.

To accomplish effective object tracking, different points of views have emerged such as using a tree-based formation [7], [8] a cluster-based formation [9] or a prediction-based method [3]-[6]. The advantage of tree based methods is in their network coverage rate and minimization of energy consumption under ideal situations. The cluster based methods aim to balance energy consumption among clusters and clusters can be formed dynamically in order to track objects. While the prediction based methods can be used to achieve successful object tracking, it calls for high rates of prediction accuracy and precision to limit energy consumption during an object tracking operation. The prediction based mechanisms can also be added onto clusterbased formations to propose a mixed or multi-phase approach for object tracking. Besides, some heuristics about predictions may also be considered to preserve energy. A short literature review on tree-based, cluster-based and 
prediction-based approaches to object tracking in WSNs can be obtained from [10].

The rest of the paper is organized as follows: In Section II, Literature Review, we discuss some of the protocols and methodologies invented for object tracking in WSNs. In Section III, Factors Affecting Object Tracking Success, the main performance metrics to evaluate the object tracking scenarios in WSNs are explained. Section IV, Proposed Network Model and Operation, clarifies the whole operation process of the sensor network in our approach to track the corresponding object within the predefined environmental boundaries. Section V, Simulation, details the simulation environment considered in our object tracking scenario. Section VI, Experiment Results, gives the results and evaluations of the experiments conducted for different network topologies. Finally, Section VII, Conclusion and Future Work, concludes the paper.

\section{LITERATURE REVIEW}

This section mainly presents a literature review in target, object, or location tracking in WSN. Some background on the use of energy in an efficient manner in general WSN applications is reviewed as well.

In the Leach protocol [9], the cluster head $(\mathrm{CH})$ in each cluster serves as the main node for data processing and transmission. This results in quick energy drain for the cluster head. To overcome this problem, they proposed randomized rotation of the cluster head role amongst the nodes of a cluster where each node takes on the cluster head role based on a threshold value determined by a probabilistic function. This threshold value $\mathrm{T}(\mathrm{n})$ is basically given by the following $[9,11,12]$ :

$$
\begin{array}{ll}
\mathrm{T}(\mathrm{n})=\frac{\mathrm{P}}{1-\mathrm{P} *\left(\mathrm{r} \bmod \frac{1}{\mathrm{P}}\right)} & \forall \mathrm{n} \in \mathrm{G} \\
\mathrm{T}(\mathrm{n})=0 & \forall \mathrm{n} \notin \mathrm{G}
\end{array}
$$

where $\mathrm{n}$ is node number for which the threshold value is to be computed, $\mathrm{r}$ is the current round, $\mathrm{P}$ is the percentage of cluster heads and $G$ is the set of nodes that have been cluster heads in the last $1 / \mathrm{P}$ rounds. The operation of the Leach protocol is divided by rounds. Each round starts with a setup phase where the clusters are organised, followed by a steady phase where the collected data is sent from the clusters to the base station. Based on the probabilistic threshold value computed by each node in distributed sensor network area, the use of energy in each cluster head node gets balanced and the network lifetime gets increased by making the nodes survive longer with probabilistic energy conservation.

Two clustering approaches for object tracking are given in [8] and [13]. In [8], a tree-structured cluster is created following the entry of an object in the monitored area. An explicit leader election mechanism is used that selects the sensor closest to the object as the $\mathrm{CH}$. Afterwards, a minimum cost tree is created that includes all the sensors within a predefined range. The tree is set to be reconfigured when distance between target and $\mathrm{CH}$ exceeds a pre-set value. In [13], in contrast to using dynamic clusters, the use of static clusters is proposed, where each cluster is activated based on detection of a target. The currently elected $\mathrm{CH}$ uses linear prediction to determine whether to keep on tracking or to switch the tracking task to another $\mathrm{CH}$.

There are some protocols that take into account quality of data for nodes which aim to reduce the amount of data being transferred in energy efficient target tracking scenarios in WSN [14]. The redundant data, which is the one collected by closely stationed nodes need only be transferred once to the cluster head. For this purpose two algorithms were proposed in [14]: Reduced Area Reporting (RARE-Area) and Reduction of Active Node Redundancy (RARE-Node). The first one limits the number of nodes taking part in object tracking by monitoring the data quality. Sensor data is assigned with a weight and the nodes that have a weight above the threshold value can participate in tracking. The second one aims to reduce the amount of redundant data by means of identifying spatial relationships between neighbouring nodes.

In Dual Prediction-based Reporting [3], both the sensor nodes and the base station make predictions about object movement to track the object. When the base station makes an error in its prediction, it is corrected by the readings of the sensor nodes.

In the Prediction-based Energy Saving scheme (PES) [4], firstly object movement is predicted to determine the suitable nodes called target nodes. After this, the selected nodes are awakened based on energy and performance metrics. Finally, a recovery mechanism is carried out if the object is missing. This recovery mechanism depends on two modes, namely ALL_NBR [4] which wakes up all the nodes surrounding the estimated route of the moving object and if this one fails, flooding recovery that wakes up all the nodes in the network in a more aggresive fashion is used.

In [10], the authors propose a distributed tracking algorithm which is run at each node of the network. This protocol distinguishes between inner nodes and the border nodes and keeps the border nodes at active state the whole time. They also propose a three level recovery system based on the positions of the nodes in the monitored area.

Another prediction-based energy-efficient target tracking protocol (PET) was proposed in [5] to derive the travelling path of the target and utilize the target's moving patterns for energy saving. Cooperation amongst sensor nodes is the key characteristic for this protocol. A linear predictor is used to predict the target's next location. As not all sensors may have useful information, sensor nodes with the best data possible are selected in order to conserve energy.

In [15] and [16], the prediction is based on the object's movement direction. In [4] and [17], the first node which senses the object wakes its one hop neighbours at first, if the object cannot be located then some more-hop neighbours are awaken, if this also fails, all the nodes are awaken at the worst case. An alternative way to choose the appropriate 
nodes to wake is selecting the nodes that have more energy for the recovery process [18].

Another study [6] proposes a prediction algorithm that is divided in clustering and prediction stages. They keep all nodes inactive except the selected $\mathrm{CH}$ nodes and when one of $\mathrm{CH}$ nodes senses an object, it becomes active and activates three more nodes using the tracker node selection algorithm. The activated nodes carry out the tracking until the current $\mathrm{CH}$ node selects the nearest $\mathrm{CH}$ node as new current $\mathrm{CH}$ node to carry out tracking.

Reference [19] proposes an energy efficient technique to predict the future movements of a mobile object using its inherited behavioural movement data patterns stored. The proposed prediction based tracking technique using sequential pattern (PTSP) offers object tracking with the efforts of a minimum number of sensor nodes in the network; meanwhile the rest of the nodes sleep to preserve the total energy.

Another target tracking approach based on a hybrid predictive model is proposed in [20] to be used in grid wireless sensor networks intrinsically. The proposed model divides the surveillance area into grids and applies a hybrid approach combining the Markov chain and the Grey Theory to predict the target path probabilistically.

A performance comparison between different kinds of tracking algorithms for tracking an object with relatively fast speed in wireless sensor networks is given in [21]. The use of cluster based versus spanning tree based target tracking algortihms are compared mainly. The corresponding clusterbased tracking algorithms involved in the comparisons use etiher a static network where the clusters are formed at deployment time, or a dynamic network where the clusters and the backbones are constructed dynamically in case of an event. Reference [21] also compares the results of adding different filtering techniques, i.e., linear, extended Kalman, and particle filters, into a proposed dynamic lookahead tree based tracking algorithm. The corresponding spanning tree based tracking algorithm is used for degrading the target miss ratio; moreover the filters are used to raise the prediction accuracy.

Another up-to-date proposal for a fast and energy efficient target tracking model based on location prediction is presented in [22]. It is pointed that the proposed method leads to a good accuracy with low energy consumption and it has low missing rates compared to linear and extended Kalman filter predictors.

\section{FACTORS AFFECTING OBJECt TRACKING SUCCESS}

With the knowledge of the approaches and mechanisms presented in Section II, this section explains the factors and the main performance metrics to evaluate the object tracking scenarios in WSNs.

The monitoring scheme deployed in a WSN setup is equally important regarding energy consumption and object tracking success rate. The sensors can be set to monitor their surroundings in scheduled monitoring mode, where all the sensor nodes are well synchronised to the base station. A dynamic clustering monitoring may be employed where sensor nodes are organised into cluster of nodes reporting to a common cluster head. A prediction based monitoring may be employed which uses a wake-up mechanism to activate specific sensors specified to be within sensing range of the object. This requires a recovery mechanism that is activated in case the object is missed. A prediction based monitoring can only be successful given the internal object location prediction algorithm is producing reliable results, otherwise the sensors will be depleted due to having to carry out a large number of recovery operations. Moreover, the object's trajectory can become the main factor affecting network lifetime, assuming an object can follow similar paths each time it enters the monitored area. There are some main factors effecting object tracking success which are explained in the following subsections.

\section{A. Energy Consumption Efficiency}

Energy consumption is directly related to how the network is designed and how it operates. Every communication made between each node in the network incurs a cost in terms of energy to nodes in the network thus should be minimised. The efficiency of the prediction algorithm as well as the mechanism used for the task of object tracking can be taken as base points to measure the energy efficiency of a sensor network.

\section{B. Accuracy of Target Tracking}

In order to keep a low probability of missing the object and for an effective target tracking application a good degree of target tracking accuracy should be achieved. This measure is also directly related to the infrastructural mechanisms used for tracking the object, i.e., using prediction based, cluster based or tree based approaches.

\section{Scalability}

Some applications may require huge numbers of sensor nodes. This can present different challenges compared to networks with smaller number of nodes. Scalability is about how well the network copes with high numbers of nodes. This study made use of a fixed number of nodes, which is relatively small to take into account scalability issues of the network.

\section{Interconnectivity}

Nodes in a sensor network need to be interconnected for the network to function properly. If there are nodes which have no route to forward packets to the access point, data collected by them will be of no use. Maximum connectivity should be achieved in the deployment stage and it should be preserved as much as possible through energy savings and congestion control.

\section{E. Network Lifetime}

Each node in a wireless sensor network is powered by a battery. This means when most of the nodes deplete their batteries the network will not be functional. Therefore it is crucial that nodes make the best use of their batteries by turning off (sleep mode) their microcontroller and transceiver when these are not needed. 


\section{PROPOSED NETWORK MODEL AND OPERATION}

WSNs typically consist of a large number of sensor nodes dispersed in a large field. This study involves simulations made using nodes distributed in $50 \times 50 \mathrm{~m}^{2}$ and $100 \times 100$ $\mathrm{m}^{2}$ fields populated with a fixed number of nodes. Comparisons between two deployment methods: grid and random were made. None of the nodes are mobile and each node in the network is set to be equal in terms of initial charge level, computation capability and communication range, namely the network only involves homogeneous sensor nodes. The sensor nodes used in the simulations have identical features to Mica2 [23] motes in terms of energy usage in active and sleep modes. Sensor nodes don't require the use of a GPS device and network lifetime diminishes when any node runs out of energy. Due to the way the ns-2 simulation is configured (area of the simulation is set in the ".tcl" scenario file) the object stays inside the boundaries of the monitored area for the duration of the simulation.

A network built for the purpose of object tracking has basically two subsets of tasks to accomplish, namely monitoring the area and reporting of the object's location to the sink node. If considered on the node level, each node is tasked to listen to its environment and report about object movement when necessary. In the most energy draining scenario, all the nodes have to be listening all the time for potential object movements. If we employ a node wise and network wise mechanism where all the nodes perform in a specific way, we can limit the time necessary to have a node in listening state. According to this specification the network can be in one of the three states: not tracking, tracking or recovering. The recovery mode works by first waking up the neighbouring nodes of the latest active nodes and goes on to wake up all the nodes in the network following a spiral route. This recovery mechanism is similar to [4] but not necessarily the same. The border nodes are the ones that are active continuously in order to make sure there is no object to track in the monitored area.

In order to track an object in the network the first requirement is to sense it and to sense the object at least some sensor nodes should be awake, for instance border nodes or randomly selected nodes. If all nodes are awake the whole time, the network lifetime shortens. If some of the nodes are sleeping in some conditions to save their power, the object could be lost again after having been found. In this situation, a recovery phase is initiated that aims to relocate the object however this leads to extra energy consumption. To achieve energy conservation and minimise object missing rate, some nodes can be awaken before the object enters their sensing territories and some nodes can be put into sleep mode after the object leaves their boundaries'. This mechanism can be triggered as follows: the active node that is sensing the object activates some other sleeping nodes. To select the appropriate nodes to put to sleep or activate requires a good prediction about the object and achieving the best possible prediction estimation means more energy can be conserved.

The nodes that have the possibility to locate the object are first predicted on the sink node. The sink node awakens a sensor node according to the initial prediction results. This is similar to the Wang's model [18], however in their model, when the predicted nodes fail to sense the object, neighborhood nodes are awakened according to the results of the genetic algorithm they use and are awakened on a one by one basis, while we take an approach in which they are awakened based on one of the three approaches we implemented.

\section{A. Prediction Model}

Our prediction algorithm is executed at the sink node, based upon the information received from tracking nodes. All the nodes depend on the sink node to determine which state they are in.

The prediction algorithm uses the well-known formula to compute velocity, which is:

$$
v=\frac{\sqrt{\left(x_{i}-x_{i-1}\right)^{2}+\left(y_{i}-y_{i-1}\right)^{2}}}{t_{i}-t_{i-1}}
$$

We use a linear prediction method as our prediction mechanism that we coded inside the ns- 2 code framework. According to the prediction algorithm results, a new set of sensor nodes are given the wake-up signal.

\section{B. Tracking Model}

When a border node detects an object in its sensing range, it initially awakens neighbour nodes so that they can carry out localization. The result of this localization is sent to the base station, which in turn uses this data to predict the location of the object. Once the newly activated nodes start sensing the object, they send it to the base station and the base station concludes that the prediction was correct and puts the previous group of nodes into sleep state. A new prediction is made in the same manner, and this loop continues until the object is lost.

The proposed tracking mechanism is based on measuring received signal strength (RSS) at the tracking nodes. RSS which is known to decrease exponentially based upon distance to the tracked object is calculated by [24]:

$$
r_{i}=a .\left\|x-x_{i}\right\|^{-\alpha}+n_{i}
$$

where $r_{i}$ is the value of the RSS in the $i^{\text {th }}$ sensor node, $a$ is the strength of the signal emitted from the target, $\mathrm{x}$ refers to the real (yet to be found) coordinates of the target, $x_{i}$ is the known coordinates of the $i^{\text {th }}$ sensor, $\alpha$ is the attenuation coefficient and lastly $\mathrm{n}_{\mathrm{i}}$ denotes the white Gaussian noise with zero-mean and variance $\sigma^{2}$.

\section{Recovery Model}

When the object is lost, the recovery mechanism is triggered which activates all the nodes starting from the neighbour nodes of the last active nodes, after activating all the nodes, if still there is no object detected, the base station concludes the object had left the area, and takes all the nodes except the border nodes back to sleep mode. 
There is a second mode of operation of the proposed system which is power saving mode. In this mode, the network acts in a way to further limit the energy consumption by increasing node deactivation frequency and decreasing number of nodes involved in the recovery process at the cost of less accurate tracking. Our simulations focused on finding the trade-off between energy conservation and accuracy of target tracking along with how the two perform for objects travelling at high and low speeds. Those two alternative modes are referred to as "Powersaving" and "Non Powersaving" while we present the measurements of our simulations via graphical results in Section VI.

The recovery mechanism we propose is analogous to the proposed model [4], in which they try to cover misprediction of object's speed and movement in their recovery phase. They propose three models: Heuristic DESTINATION, Heuristic ROUTE and Heuristic ALL NBR. Each of these has different energy efficiencies. The first one assumes the speed and direction of the object is predicted correctly and wakes up one node on the predicted path. The second one assumes the speed of the object is mispredicted and the current node informs nodes on the predicted path. It assumes the direction is correctly predicted. The third one assumes both the speed and the direction of the object are mispredicted and the current node informs the neighboring nodes that surround the route. The sink node, wakes up a node (current node) based on the prediction result, and when this node loses track of the object, it first informs a neighbour node and based on the result it gets from the other node, it either informs the sink node or doesn't. If it informs the sink node, this shows that this node also failed to sense the object, if it doesn't inform, it means the node sensed the object and there was no need for further recovery. The node that sensed the object now becomes the current node.

The difference of our model compared to the energy conservative approach proposed in [4] is that the prediction is done on the sink node and the sink node determines the current node except in situations where the object is lost temporarily and found (neighbour node becomes the current node) or when a node detects a new object by chance in the area. In addition to this, we adapt a more aggressive approach for recovery where we begin to wake up all the nodes as well the ones on the predicted path.

The difference we propose compared to the recovery mechanism of [4] is that, we define a spiral route that begins from the closest neighbour of the last current node (nearest place where the object was lost) that continues to wake up all the nodes in the network until the object is found. We aim to minimise the time it takes to wake up all the nodes in this manner and put them to sleep if the object had already left the area. We also aim to make transitions between these states (prediction, tracking, recovery) as fast as possible in order to make sure the object is tracked for the time it is inside the network and also to increase energy efficieny.

\section{V.SIMULATION}

The IEEE 802.15.4 [25] medium access control (MAC) protocol is used for our scenario implementation on ns-2.34 [26]. Different from the IEEE 802.11 [27] protocol which is used for WLAN networks, IEEE 802.15.4 protocol is a low tier, ad hoc, terrestrial, wireless standard for wireless networks and other ad hoc networks such as WSNs. The main simulation parameters used in our different scenarios are given in Table I below.

TABLE I.

SiMULATION PARAMETERS

\begin{tabular}{ll} 
Description & Value \\
\hline Simulation Environment & ns-2.34 \\
PHY-MAC Layer & IEEE 802.15 .4 \\
Field Size & $50 \mathrm{~m}$ x $50 \mathrm{~m}$, \\
& $100 \mathrm{~m}$ x $100 \mathrm{~m}$ \\
Tracking Node Number & 21 \\
Sink Node Number & 1 \\
Sensor Node Deployment & Uniformly Distributed, \\
& Randomly Distributed \\
Energy Consumption (Active & \\
Mode) & $8 \mathrm{~mA}$ \\
Energy Consumption (Sleep & \\
Mode) & $<15 \mathrm{uA}$ \\
Communication Range & $40 \mathrm{~m}$ \\
Sensing Range & $\sim 15 \mathrm{~m}$ \\
Velocity of Target & $5 \mathrm{~m} / \mathrm{s}-16 \mathrm{~m} / \mathrm{s}$ \\
Duration of Simulations & $200 \mathrm{~s}$ \\
Number of Trials & 5 \\
Number of Tracked Target & 1 \\
\hline
\end{tabular}

A sensor network consisting of 21 tracking nodes uniformly distributed in a field of $50 \times 50 \mathrm{~m}^{2}$ and a sink node at the lower left-hand side is considered. All the sensor nodes are homogeneous and immobile. The topological deployment of the nodes can be seen below in Fig. 1. The tracked object is denoted by a full circle and the sink node is

TABLE II.

State Transition Table OF THE FSM

\begin{tabular}{|c|c|c|c|c|c|c|}
\hline & Time_Out & Wake_Up & $\begin{array}{c}\text { RSS }> \\
\text { Threshold }\end{array}$ & RSS < Threshold & $\begin{array}{l}\text { On_Predicted } \\
\text { Path }\end{array}$ & $\begin{array}{l}\text { Object_ } \\
\text { Area }\end{array}$ \\
\hline SLEEP & - & SENSE & - & - & - & - \\
\hline SENSE & SLEEP & - & DETECTED & - & - \\
\hline DETECTED & - & - & DETECTED & WAIT_MESSAGE & - \\
\hline $\begin{array}{c}\text { WAIT_ } \\
\text { MESSAGE }\end{array}$ & - & - & - & - & SENSE & - \\
\hline RECOVERY & - & - & DETECTED & - & - \\
\hline
\end{tabular}


designated as a pentagon to differentiate it from the ordinary tracking sensor nodes. Sink node, in our proposal, is responsible for running the prediction algorithm and activating the sensor nodes according to the prediction results.

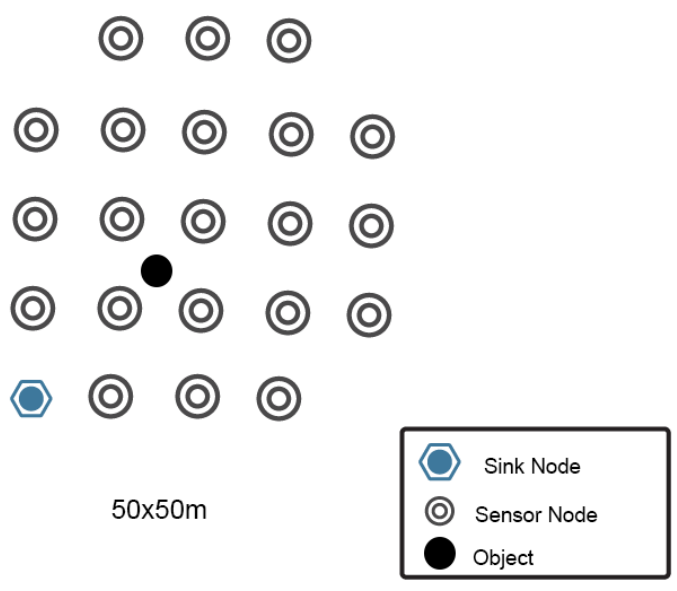

Fig. 1 Main Network Topology

In preparation of the simulation, modifications to the ns- 2 code base and implementations of additional functions were defined. An application layer based on the proposed scenario was implemented on top of ns-2.34's IEEE 802.15.4 MAC layer. For transport layer, we modified the message agent which sends packets in similar way to UDP. The most important function is the process message which processes incoming messages and changes the states of nodes. We completely rewrote the message processing functions along with all the functions on the application layer.

Each node is simulated as having five different states during network operation. It is drawn as a finite state machine (FSM) and coded inside the ns-2 code base along with the prediction algorithm. The finite state machine is depicted in Fig. 2, a corresponding state transition table is provided in Table II and detailed descriptions of each state and message used are given below.

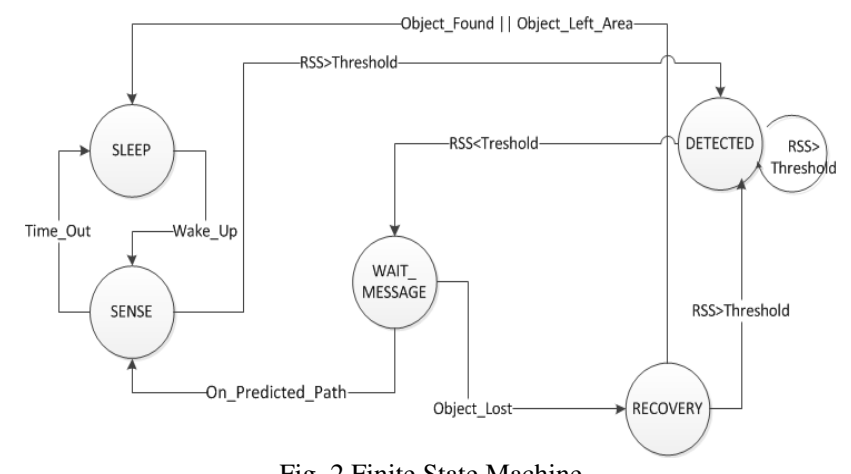

Fig. 2 Finite State Machine

1) States:

SLEEP: In this state, sensor node does not receive or send any messages and keeps energy efficiency at maximum. In order to receive messages from the network, sensor nodes change state to SENSE periodically.
SENSE: In sense state, a sensor node can send and receive messages and periodically changes its state to SLEEP. The sensor nodes selected by the prediction algorithm are in this state and they are expected to have the object in close proximity. If a sensor node senses an object in this state, it changes its state to DETECTED.

DETECTED: Sensor nodes stay in this state as long as their RSS value is greater than the threshold value. If a sensor node cannot sense the object anymore then it changes its state to wait message.

WAIT_MESSAGE: In this state, sensor nodes wait for the sense message of the next node on the predicted path. If it receives On_Predicted_Path message then it changes its state to SENSE and if it receives Object_Lost message it changes to RECOVERY.

RECOVERY: In recovery state, unless Object_Found or Object_Left_Area messages aren't received from the sink node, sensor node stays in this state. If RSS value is greater than threshold value then it changes its state to DETECTED and sends data about object's position.

2) Messages:

Time_Out: When the predefined timeout value in a node runs out the node is given the timeout signal.

Wake_Up: Sink node sends this message to nodes in the predicted path.

On_Predicted_Path: This message is for nodes that no longer sense the object but may still be on the predicted path and be required to continue to sense.

RSS>Threshold: When the RSS value of a node is above the threshold value it means it can start tracking the object.

RSS<Threshold: When the RSS value of a node is below the threshold value it means it no longer tracks the object.

Object_Lost: This message is sent to specified nodes depending on the stage of the recovery mode to inform that recovery mode is initiated.

Object_Found: This message is sent in recovery mode to searching nodes which were unsuccessful in sensing the object.

Object_Left_Area: This message is sent to all sensor nodes if the recovery mode fails to recover the object in a specified time.

\section{EXPERIMENT RESULTS}

Different scenarios were setup and each one involved objects with two different speeds: $5 \mathrm{~m} / \mathrm{s}$ and $16 \mathrm{~m} / \mathrm{s}$. The first one stands for low speed objects and the latter stands for high speed objects respectively. The main purpose was to compute average energy loss and average recovery time after measuring their absolute values by running each simulation scenario for 5 times. The experiments were also conducted with medium speeds, i.e., $8 \mathrm{~m} / \mathrm{s}$ and $12 \mathrm{~m} / \mathrm{s}$.

At the end of each simulation, data on total energy spent and time lengths of recovery for each object were obtained. The data gathered from the simulation was analysed and put on graphs for better visualization. The object moves following linear paths and bounces off at area boundaries. For localization, trilateration [28] is used to determine target 
position along with RSS value to measure distance to the target.

Simulations were mostly conducted on one (main) topology and later on the simulation was extended to include three more different topologies. The decision to extend the simulation emerged from the need to compare obtained results with results from a different deployment model (random). The main topology used is given in Fig. 1. This first topology (referred to as Topology1) includes nodes that are uniformly distributed in the area.

In the second topology (Topology2), the field was increased to an area of $100 \times 100 \mathrm{~m}^{2}$, increasing the length between nodes but keeping the same formation as the first topology. The third topology (Topology3) covers randomly distributed nodes in a $50 \times 50 \mathrm{~m}^{2}$ field. The last topology (Topology4) involves nodes randomly distributed in a field of $100 \times 100 \mathrm{~m}^{2}$.

By conducting various simulation experiments, the aim was to measure the success of our approach and to compare the results for low speed and high speed objects. In addition, two modes of operation have been taken into consideration, namely powersaving and non powersaving modes for the operation of the network. Each node in the network has the same starting energy of 20 Joules at the beginning of any simulation. The average remaining energy per node, average recovery delay, and packet delivery ratio results of simulations conducted on Topology1 are given in Fig. 3, Fig. 4, and Fig. 5.

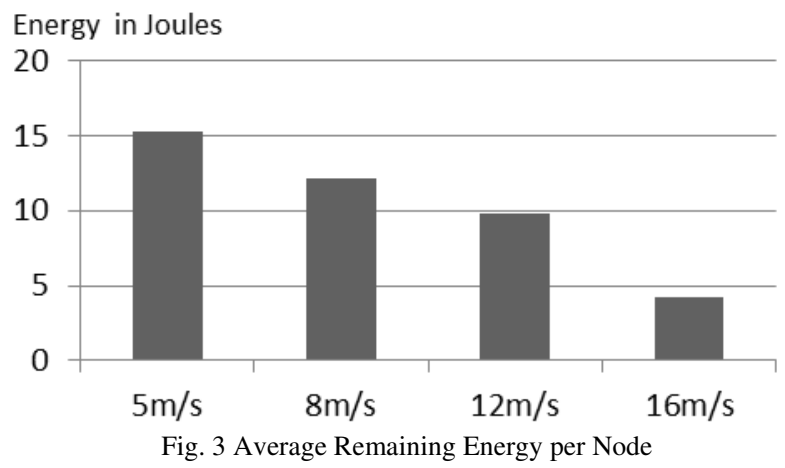

In Fig. 3, the displayed energy levels regarding the related speed values of the moving object belong to the average remaining energy per node. The remaining energy value of each node is measured at the end of each simulation and the corresponding average remaining energy value per node is calculated for the whole network. The average values of the calculation results for 5 repeated simulations are given in Fig. 3. The relationship between object speed and the total remaining energy of all the nodes can be seen in Fig. 3, object's speed has a direct relationship to energy consumption levels. This is due to increased object loss rates for objects travelling at high speeds. As the recovery frequency increases, so does the total energy consumption. Taking the results shown in Fig. 3 as an example, for an object travelling at $16 \mathrm{~m} / \mathrm{s}$ the average remaining energy drops as low as 4.2 Joules, where the energy consumption in the whole network is greater than the other cases for slower speeds.

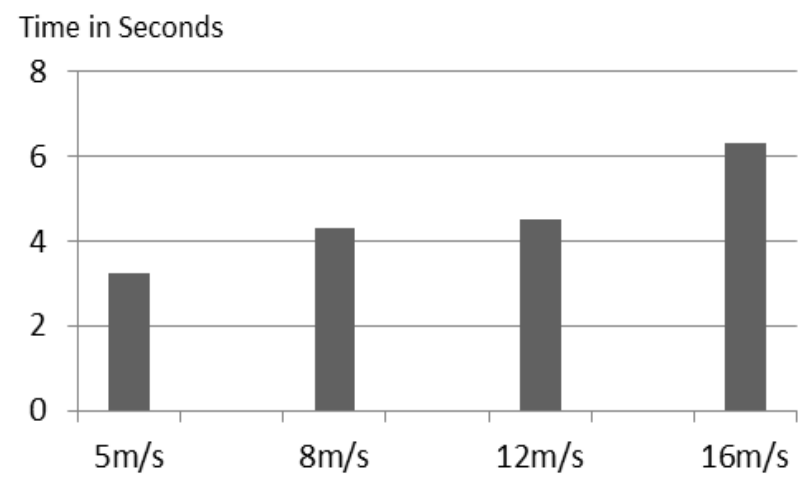

Fig. 4 Average Recovery Time

In Fig. 4, we can see the direct correlation between object speed and average recovery time in seconds. For objects travelling at $5 \mathrm{~m} / \mathrm{s}$ average recovery time is as low as 3.23 seconds and for objects travelling at $16 \mathrm{~m} / \mathrm{s}$ the corresponding duration is 6.32 seconds which is significantly higher compared to the former. For objects travelling at $8 \mathrm{~m} / \mathrm{s}$ and $12 \mathrm{~m} / \mathrm{s}$ average recovery times of 4.32 and 4.39 seconds are observed respectively.

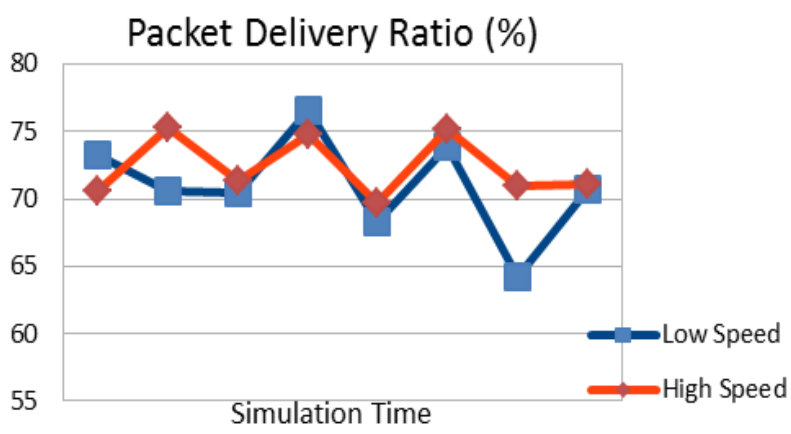

Fig. 5 Ratio of Packets Successfully Delivered

Fig. 5 shows the packet delivery success ratio in the network. Since the prediction in our model is done on the sink node, the tracking object's location data is delivered to the sink when it's detected by any node or group of nodes. Meanwhile, the data may be lost in the network due to the interference and collision of the packets. Besides, IEEE 802.15.4 PHY and MAC layers inherit the packet drops of the wireless media. To us, on average a $72 \%$ success ratio for the packet delivery seen in Fig. 5 makes sense regarding the collisions intrinsically available in the wireless channel. We also deduce from Fig. 5 that the oscillation of the packet delivery success during the whole simulation is due to random variation of the number of collided packets at random times.

By adding three other topologies, as mentioned before, we extended our study; Fig. 6 and Fig. 7 show the comparison of results obtained for objects travelling at low speeds and high speeds, respectively. The aforementioned "Powersaving" and "Non Powersaving" terms in Fig. 6 and Fig. 7 refer to the two different running modes of the system explained in Subsection C (Recovery Model) of Section IV. 
When running in the non powersaving mode, the system does its best to track and detect the object and uses its all resources to achieve this task. This means the system rigorously awakens all the nodes if necessary regarding the procedures of the recovery algorithm used. On the other hand, if the system is adjusted to run in powersaving mode, the recovery task will be switched into a way to limit the energy consumption at the cost of less accurate tracking. The simulations of which the results are depicted in Fig. 6 and Fig. 7 focus on finding the trade-off between energy conservation and accuracy of target tracking along with how the two perform for objects travelling at high and low speeds.

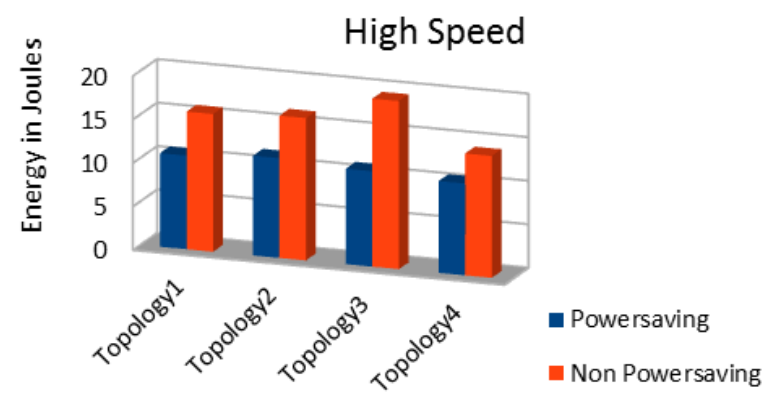

Fig. 6 Average Energy Consumption for High Speed Object

The graph in Fig. 6 shows the amounts of average energy consumed per node with different topological simulation setups for objects travelling at high speeds. Regarding each different topology, energy preservation algorithm makes a considerable difference in terms of energy savings in the network. The disadvantage of powersaving mode compared to non powersaving mode is reduced tracking accuracy. All four topologies have similar energy consumption levels for powersaving mode, whereas for non powersaving mode, energy consumption is more varied across different topological setups. Topology3 has the highest amount of total energy consumption for non powersaving mode.

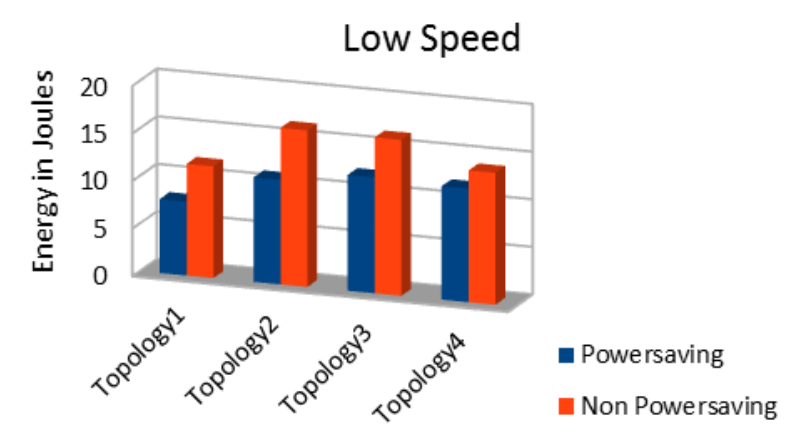

Fig. 7 Average Energy Consumption for Low Speed Object

The top-view of the graph in Fig. 7 seems similar with the one in Fig. 6, except that the exact results are different for the case that the object moves at a low speed. According to Fig. 7, all eight different simulation scenarios (four topologies within two operating modes) show varied energy levels. The minimum energy consumption in total for both running modes is observed in Topology1, whereas the maximum energy consumption is measured in Topology 3 among the eight different scenarios.

The disadvantage of powersaving mode is its reduced tracking accuracy due to the adjusted awakening algorithm involved in the recovery model to limit energy consumption. As a result of the trade-off observed between tracking accuracy and energy consumption, the advantage of powersaving mode is significantly less energy consumption. As evident in Fig. 6 and Fig. 7, regarding the energy consumption levels, powersaving mode has a distinctive advantage both for objects travelling at high speeds and low speeds. It can also be inferred from Fig. 6 and Fig. 7 that non powersaving scenarios in which the tracking object moves with high speeds usually consume more energy than the non powersaving scenarios involving a tracking object with low speeds. This is mainly because of the higher number of recovery stages incurred in high speed scenarios. By using powersaving mode in such high speed scenarios, we can limit energy consumption to the levels observed in low speed scenarios.

\section{CONCLUSION AND FUTURE WORK}

This paper presents a network simulation based study aiming to answer the question of "how can energy efficiency be improved in an OTSN through implementing various scenarios for object tracking". Having presented our initial work at the national conference of Academic Computing in Turkey [29], we extend our work to include the proposed network operation and simulation results. This network setup was simulated in ns-2.34 and associated graphs of obtained results are given.

Different scenarios were considered where target speeds and moving patterns vary. The main focus has been on energy conservation which has been mostly achieved by reducing the average energy consumed by sensor nodes.

As future work, we wish to improve our tracking method to track multiple objects simultaneously. We also think that, a dynamic clustering of nodes before or after the prediction phase may make a difference in terms of improving tracking success and energy efficiency.

\section{ACKNOWLEDGMENT}

This project initially started as a senior project at Izmir University of Economics involving six people in total. We would like to thank to Utkan Surgevil, Yetkin Hafizoglu and Nihal Pacaman for their efforts.

\section{REFERENCES}

[1] I. F. Akyildiz, W. Su, Y. Sankarasubramaniam, and E. Cayirci, "Wireless sensor networks: a survey," Computer Networks, vol. 38, pp. 393-422, 2002, http://dx.doi.org/10.1016/S1389-1286(01)00302-4

[2] H. Karl and A. Willig, Protocols and Architectures for Wireless Sensor Networks: John Wiley \& Sons, 2005, http://dx.doi.org/10.1002/0470095121

[3] Y. Xu, J. Winter, and W.-C. Lee, "Dual prediction-based reporting for object tracking sensor networks," in The First Annual International Conference on Mobile and Ubiquitous 
Systems: Networking and Services, MOBIQUITOUS 2004, Aug. 2004, pp. 154-163, http://dx.doi.org/10.1109/ MOBIQ.2004.1331722

[4] Y. Xu, J. Winter, and W.-C. Lee, "Prediction-based strategies for energy saving in object tracking sensor networks," in Proceedings of IEEE International Conference on Mobile Data Management, 2004, pp. 346357, http://dx.doi.org/10.1109/MDM.2004.1263084

[5] M. Z. A. Bhuiyan, G.-J. Wang, L. Zhang, and Y. Peng, "Prediction-based energy-efficient target tracking protocol in wireless sensor networks," Journal of Central South University of Technology, vol. 17(2), pp. 340-348, 2010, http://dx.doi.org/10.1007/s11771-010-0051-1

[6] F. Deldar and M. H. Yaghmaee, "Designing an energy efficient prediction-based algorithm for target tracking in wireless sensor networks," 2011 International Conference on Wireless Communications and Signal Processing (WCSP), Nov. 2011, pp. 1-6, http://dx.doi.org/10.1109/ WCSP.2011.6096835

[7] H. T. Kung and D. Vlah, "Efficient location tracking using sensor networks," 2003 IEEE Wireless Communications and Networking, WCNC 2003, March 2003, pp. 1954-1961 vol. 3, http://dx.doi.org/10.1109/WCNC.2003.1200686

[8] W. Z. W. Zhang and G. C. G. Cao, "DCTC: dynamic convoy tree-based collaboration for target tracking in sensor networks," IEEE Transactions on Wireless Communications, vol. 3(5), pp. 1689-1701, 2004, http://dx.doi.org/ 10.1109/TWC.2004.833443

[9] W. R. Heinzelman, A. Chandrakasan, and H. Balakrishnan, "Energy-efficient communication protocol for wireless microsensor networks," in Proceedings of the 33rd Annual Hawaii International Conference on System Sciences, Jan. 2000, http://dx.doi.org/10.1109/HICSS.2000.926982

[10] S. P. M. Tran and T. A. Yang, "OCO: Optimized communication \& organization for target tracking in wireless sensor networks," in Proceedings of the IEEE International Conference on Sensor Networks, Ubiquitous, and Trustworthy Computing (SUTC'06), June 2006, pp. 428435, http://dx.doi.org/10.1109/SUTC.2006.1636209

[11] W. B. Heinzelman, A. P. Chandrakasan, and H. Balakrishnan, "An application-specific protocol architecture for wireless microsensor networks," Wireless Communications, IEEE Transactions on, vol. 1(4), pp. 660670, 2002, http://dx.doi.org/10.1109/TWC.2002.804190

[12] M. J. Handy, M. Haase, and D. Timmermann, "Low energy adaptive clustering hierarchy with deterministic cluster-head selection," in Mobile and Wireless Communications Network, 2002. 4th International Workshop on, 2002, pp. 368-372, http://dx.doi.org/10.1109/MWCN.2002.1045790

[13] H. Yang and B. Sikdar, "A protocol for tracking mobile targets using sensor networks," in Proceedings of 2003 IEEE International Workshop on Sensor Network Protocols and Applications, May 2003, pp. 71-81, http://dx.doi.org/10.1109/SNPA.2003.1203358

[14] M. Guo, E. Olule, G. Wang, and S. Guo, "Designing energy efficient target tracking protocol with quality monitoring in wireless sensor networks," The Journal of Supercomputing, vol. 51(2), pp. 131-148, 2010, http://dx.doi.org/10.1007/s11227-009-0278-5

[15] F. Zhao, J. Shin, and J. Reich, "Information driven dynamic sensor collaboration," IEEE Signal Processing Magazine, vol. 19(2), pp. 61-72, 2002, http://dx.doi.org/10.1109/ 79.985685

[16] P. V. Pahalawatta, T. N. Pappas, and A. K. Katsaggelos, "Optimal sensor selection for video-based target tracking in a wireless sensor network," in 2004 International Conference on Image Processing, ICIP '04, Oct. 2004, pp. 3073-3076 Vol. 5, http://dx.doi.org/10.1109/ICIP.2004. 1421762

[17] Z. Guo, M. Zhou, and L. Zakrevski, "Optimal tracking interval for predictive tracking in wireless sensor network," Communications Letters, IEEE, vol. 9(9), pp. 805-807, 2005, http://dx.doi.org/10.1109/LCOMM.2005.1506709

[18] X. Wang, L. Ding, D. Bi, and S. Wang, "Energy-efficient optimization of reorganization-enabled wireless sensor networks," Sensors, vol. 7(9), pp. 1793-1816, 2007, http://dx.doi.org/10.3390/s7091793

[19] S. Samarah, M. Al-Hajri, and A. Boukerche, "A predictive energy-efficient technique to support object-tracking sensor networks," IEEE Transactions on Vehicular Technology, vol. 60(2), pp. 656-663, 2011, http://dx.doi.org/ 10.1109/TVT.2010.2102375

[20] Y.-L. Chen, Y.-C. Lin, and T.-C. Sun, "A prediction scheme for object tracking in grid wireless sensor networks," in Seventh International Conference on Innovative Mobile and Internet Services in Ubiquitous Computing (IMIS), July 2013, pp. 360-364, http://dx.doi.org/10.1109/IMIS.2013.67

[21] A. Alaybeyoglu, A. Kantarci, and K. Erciyes, "A dynamic lookahead tree based tracking algorithm for wireless sensor networks using particle filtering technique," Computers \& Electrical Engineering, vol. 40(2), pp. 374-383, 2014, http://dx.doi.org/10.1016/j.compeleceng.2013.06.014

[22] M. Mirsadeghi and A. Mahani, "Energy efficient fast predictor for WSN-based target tracking," Annals of Telecommunications, pp. 1-9, 2014, http://dx.doi.org/ 10.1007/s12243-014-0430-y

[23] Mica2 Datasheet,

http://www.eol.ucar.edu/isf/facilities/isa/internal/CrossBow/ DataSheets/mica2.pdf . (Last accessed: March 2014)

[24] W.-P. Chen, J. C. Hou, and L. Sha, "Dynamic clustering for acoustic target tracking in wireless sensor networks," IEEE Transactions on Mobile Computing, vol. 3(3), pp. 258-271, 2004, http://dx.doi.org/10.1109/TMC.2004.22

[25] Wireless medium access control and physical layer specifications for low-rate wireless personal area networks. IEEE Standard, 802.15.4-2003, May 2003. ISBN 0-73813677-5.

[26] Network Simulator 2 (ns-2), www.isi.edu/nsnam/ns/. (Last accessed: March 2014)

[27] Wireless medium access control and physical layer specifications for low-rate wireless personal area networks. IEEE Standard, 802.11-2007, June 2007. ISBN 0-73815656-6.

[28] B. Krishnamachari, S. B. Wicker, and R. Bejar, "Phase transition phenomena in wireless ad hoc networks," in IEEE Global Telecommunications Conference, GLOBECOM '01, Nov. 2001, pp. 2921-2925 vol.5, http://dx.doi.org/ 10.1109/GLOCOM.2001.965963

[29] G. Peynirci, M. Gürgen, İ. Korkmaz, Y. Hafizoğlu, U. Sürgevil, and N. Paçaman, "An object tracking scenario using wireless sensor networks," presented at the Academic Computing, Turkey, 2010. 